\title{
A!
}

This is an electronic reprint of the original article.

This reprint may differ from the original in pagination and typographic detail.

Tzarouchis, Dimitrios C.; Sihvola, Ari

\section{Polarizability of Radially Inhomogeneous Subwavelength Spheres}

Published in:

Physical Review Applied

DOI:

10.1103/PhysRevApplied.10.054012

Published: 06/11/2018

Document Version

Publisher's PDF, also known as Version of record

Please cite the original version:

Tzarouchis, D. C., \& Sihvola, A. (2018). Polarizability of Radially Inhomogeneous Subwavelength Spheres.

Physical Review Applied, 10(5). https://doi.org/10.1103/PhysRevApplied.10.054012

This material is protected by copyright and other intellectual property rights, and duplication or sale of all or part of any of the repository collections is not permitted, except that material may be duplicated by you for your research use or educational purposes in electronic or print form. You must obtain permission for any other use. Electronic or print copies may not be offered, whether for sale or otherwise to anyone who is not an authorised user. 


\title{
Polarizability of Radially Inhomogeneous Subwavelength Spheres
}

\author{
Dimitrios C. Tzarouchis ${ }^{1,2, *}$ and Ari Sihvola ${ }^{1}$ \\ ${ }^{1}$ Department of Electronics and Nanoengineering, Aalto University, Maarintie 8, 02150 Espoo, Finland \\ ${ }^{2}$ Electrical and Systems Engineering Department, University of Pennsylvania, 200 South and 33rd Street, \\ Pennsylvania, 19104-6314 Philadelphia, USA
}

(Received 12 April 2018; revised manuscript received 8 August 2018; published 6 November 2018)

\begin{abstract}
In this work the polarizability of a subwavelength core-shell sphere is considered, where the shell exhibits a radially inhomogeneous permittivity profile. The mathematical treatment of the electrostatic polarizability is formulated in terms of the scattering potentials and the corresponding scattering amplitudes. As a result, a generalized expression of the polarizability is presented to be dependent of the radial inhomogeneity function. The extracted general model is applied for two particular cases, i.e., a power-law profile and a new class of permittivity profiles that exhibit exponential radial dependence. The proposed analysis quantifies in a simple manner the inhomogeneity effects, allowing the direct implementation of naturally or artificially occurring permittivity inhomogeneities for a wide range of applications within and beyond the metamaterial paradigm. Specifically, a special case of symmetric-antisymmetric resonant plasmonic degeneracy is identified and shown for the case of a core-shell sphere with a power-law permittivity profile. This degeneracy could be used for the experimental identification of inhomogeneity-induced effects or for applications where a strong coupling resonant regime is required. Furthermore, the described analysis opens avenues towards the phenomenological and first-principles modeling of the electrodynamic scattering effects for graded-index plasmonic particles at the nanoscale. Finally, such a description can be readily used either for the benchmarking of novel computational methods incorporating inhomogeneous materials or for inverse scattering purposes.
\end{abstract}

DOI: 10.1103/PhysRevApplied.10.054012

\section{INTRODUCTION}

Electromagnetic scattering by a subwavelength sphere is a canonical and fundamental problem found in the core of many areas such as RF and optical engineering, bioengineering, and material sciences [1-3]. The significance of this problem reaches from conventional applications, such as sensing [2] and energy harvesting [4], towards more exotic ones, such as invisibility cloaks [5], super scatterers [6], and optical energy localization [7]. Studies on subwavelength spherical scatterers unveiled several fundamental aspects of the resonant scattering of a sphere, a testbed for extracting physical intuition for a plethora of phenomena in physics like the plasmon hybridization and Fano resonances on single scatterers [8-10].

This universality emerges, perhaps, from the fact that the scattering response of a small dielectric sphere can be rigorously quantified by a (normalized) polarizability expression [11]:

$$
\alpha=3 \frac{\varepsilon_{1}-\varepsilon_{h}}{\varepsilon_{1}+2 \varepsilon_{h}},
$$

where $\varepsilon_{1}$ is the permittivity of the sphere embedded in a host medium of permittivity $\varepsilon_{h}$. This simple expression

\footnotetext{
*dimitrios.tzarouchis@aalto.fi
}

conveys a wealth of physical phenomena, such as the position and the width of the localized surface plasmon resonance (LSPR) or plasmonic resonance $\left(\varepsilon_{1}=-2 \varepsilon_{h}\right)$. The very same expression can be found in a large number of studies on the modeling of small resonant elements with dipolelike radiation [12-15]. Therefore, revisiting and refining the context of this expression can potentially have an effect on a wide range of disciplines.

In this work, the concept of the polarizability is imposed to a more general case of a sphere with a radially inhomogeneous (graded-index, or RI) permittivity profile. These kinds of profiles occur either naturally [16], or as a result of sophisticated engineering processes $[17,18]$. However, this category can include even simpler structures, such as core-shell spheres, which are nothing but inhomogeneous spheres with a stepwise permittivity profile. Hence, by studying the properties of RI spheres we can expand our current understanding on the effects of partially or continuously inhomogeneous scatterers.

The concept of an RI profile has a history. In optical and radio engineering, the Luneburg, Eaton, and Maxwell fisheye lenses are characteristic examples of the utilization of inhomogeneity for tailoring the electromagnetic scattering response [19-21]. These first examples were initially analyzed within the geometrical optics approximations. 
Quickly after these problems were reformulated as a classical boundary value problem using Maxwell equations, and rigorous remedies were available. Aden and Kerker, in their seminal work [22] delivered an $\grave{a}-l a$ Lorenz-Mie solution to the problem of a core-shell structure, while Wait [23] generalized the stepwise homogeneous problem for spheres with $n$ layers. In this way, any RI profile can be constructed by a stratified sphere with variable permittivities for each layer. Undoubtedly, this robust brute-force methodology solves the required scattering problem, however, without offering physical intuition on the involved mechanisms.

An alternative treatment for the RI problem is the invariant imbedding technique [24-26]. This technique treats the step-homogenous problem in its infinite layer limit reformulating the original scattering problem to a problem that satisfies a nonlinear Riccati equation. Subsequently, the resulting nonlinear equation is numerically evaluated for a given permittivity profile. Hence the invariant imbedding technique can be categorized as a semianalytical approach. Furthermore, the RI problem has been attacked by several different volume-based numerical philosophies (see for example [27,28]). Apparently, all the aforementioned remedies are used mostly for designing the scattering behavior of RI particles. The main drawback of these methods is that they rarely offer any physical insights on the involved scattering mechanisms and their particular characteristics.

One possible treatment that restores the physical intuition is obviously the exact solution of the corresponding boundary value problem, and the formulated differential equation (second order) of the radial function, for a given graded-index profile. A comprehensive overview of the available exact solutions for the electrodynamic problem up to the late 1960s can be found in [29], while more recent works are listed in [30]. For instance, the exact solution for a sphere with a Luneburg profile has been given by Tai [31], where the formulated radial differential equation is satisfied by a hypergeometric function, while Westcott explored the available exact wave solutions for spherical stratified media [32].

The corresponding problem of an RI subwavelength sphere in the electrostatic (Rayleigh) limit and the available solutions of the inhomogeneous Laplace equation have also been reviewed in the past [33]. More recent works on different RI profiles with power-law [34], linear [35], and polynomial profiles (with some convergence restrictions) [36] brought into light some features of the deeply subwavelength RI problem; a systematic study categorizing all the available analytical solutions for the electrostatic problem is still missing.

Inspired by the above developments and realizing the remaining gaps, we revisit the concept of polarizability towards a generalized description that incorporates the effects of the inhomogeneous permittivity. Under this fresh perspective we revisit the known case of a power-law profile and explore its scattering peculiarities. The proposed model is further expanded towards a new family of permittivity profiles with an exact solution, i.e., exponentially radial profiles. Results on their resonant spectrum reveal the existence of several scattering characteristics, such as shifted plasmonic resonances and peculiar scattering degeneracies for extreme permittivity values. Specifically, a particular type of symmetric-antisymmetric resonant plasmonic scattering degeneracy is identified, as a result of the shell inhomogeneity on a core-shell sphere.

The presented results can potentially stimulate further discussions about the theory of RI scatterers, their particular functionalities, and open fertile grounds toward their experimental implementation on modern energy control and harvesting applications. The analysis concludes by reflecting on how the presented results can be used for scatterers with naturally or artificially occurring inhomogeneities, such as particles exposed to temperature and pressure gradients, irregular shaped and ligand molecules, and extremely small particles exhibiting smooth boundaries. The generalization of the polarizability can also be particularly useful in connection with inverse scattering problems [37], where the main objective often is the identification of unique material inhomogeneities encoded in the signatures of the observed spectra.

\section{THEORY}

Let us assume a sphere (Fig. 1, with subscript 0 or $h$ for external (host), 1 for shell, and 2 for core domain) of radii $r_{1}$ and $r_{2}$, subject to a uniform ( $z$-directed) electrostatic field causing a scattering potential of dipolar character, i.e.,

$$
\Phi_{0}(r, \theta)=\left(-E_{0} r+\frac{B_{0}}{r^{2}}\right) \cos \theta .
$$

Since the core region consists of a homogeneous material we have

$$
\Phi_{2}(r, \theta)=A_{2} r \cos \theta,
$$

while the potential in the shell region can be written as

$$
\Phi_{1}(r, \theta)=f(r) \cos \theta
$$

assuming an arbitrary radial function $f(r)$. The expressions of the scattered (external, region 0 ) and the internal (core, region 2) field $(\mathbf{E}=-\nabla \Phi)$ are divergenceless, and satisfy the other requirements of the corresponding physical problem, i.e., the scattered field vanishes at large distances and has no singularities at the origin [38]. In a similar manner, we also require divergenceless electric flux density at the 


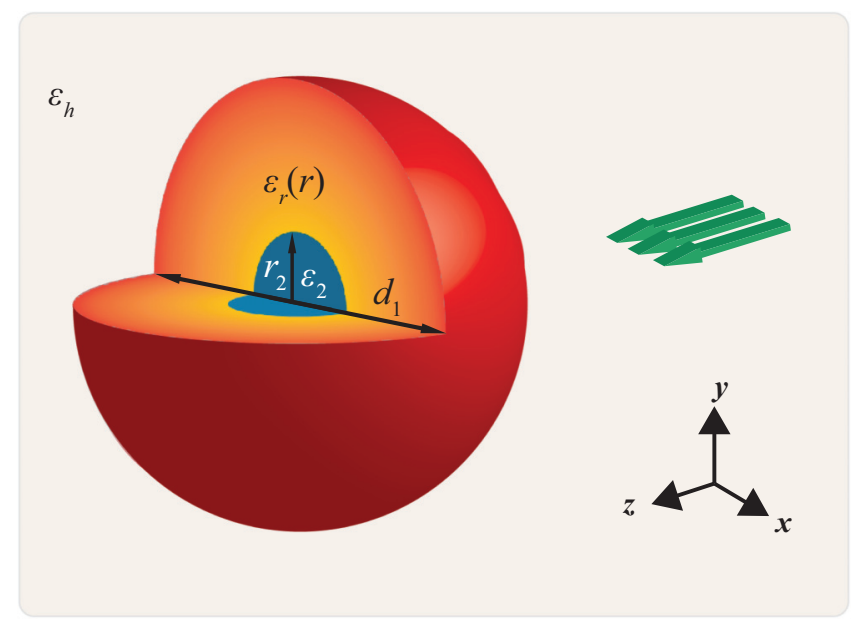

FIG. 1. A radially inhomogeneous sphere of diameter $d_{1}=2 r_{1}$ and radially inhomogeneous permittivity $\varepsilon_{r}(r)$, with an internal homogeneous core of radius $r_{2}$ and permittivity $\varepsilon_{2}$ immersed in a host medium $\left(\varepsilon_{h}\right)$ subject to a $z$-polarized plane wave (constant excitation field in the long-wave approximation).

inhomogeneous region (shell, region 1), viz.,

$$
\nabla \cdot \mathbf{D}_{1}=-\nabla \cdot\left[\varepsilon_{r}(r) \nabla \Phi_{1}(r, \theta)\right]=0,
$$

resulting in the following ordinary differential equation (ODE):

$$
f^{\prime \prime}(r)+\left[\frac{2}{r}+\frac{\varepsilon_{r}^{\prime}(r)}{\varepsilon_{r}(r)}\right] f^{\prime}(r)-\frac{2}{r^{2}} f(r)=0 .
$$

The main focus here is to study the cases where Eq. (6) obtains a closed form solution, hence extracting an analytical expression for the unknown scattering amplitudes by solving the formulated boundary value problem. For these cases one can express the radial function as

$$
f(r)=A_{1} A(r)+B_{1} B(r),
$$

where $A(r)$ and $B(r)$ are two linearly independent solutions of the radial function. For instance, the case of a homogeneous profile gives the well-known expressions $A(r)=r$ and $B(r)=1 / r^{2}$.

The extension of the analysis including higher-order multipoles can be done in a similar manner as described in $[34,35,38]$. In this work, we concentrate only on the main dipole contribution since in many practical cases subwavelength spherical inclusions induce primarily a dipole field for a given plane-wave excitation. This is due to the symmetry of the excitation. Different sources, i.e., with different symmetries such as dipoles or focused beams, couple efficiently also with higher-order modes. For these cases a higher-order analysis and treatment is required, a subject left here for future investigations.
The electric fields for each domain are

$$
\begin{aligned}
\mathbf{E}_{0}(r, \theta)= & E_{0} \mathbf{u}_{z}+\frac{B_{0}}{r^{3}}\left(2 \cos \theta \mathbf{u}_{r}+\sin \theta \mathbf{u}_{\theta}\right) \\
\mathbf{E}_{1}(r, \theta)= & -\left[A_{1} A^{\prime}(r)+B_{1} B^{\prime}(r)\right] \cos \theta \mathbf{u}_{r} \\
& +\left[A_{1} A(r)+B_{1} B(r)\right] \frac{\sin \theta}{r} \mathbf{u}_{\theta} \\
\mathbf{E}_{2}(r, \theta)= & -A_{2} \mathbf{u}_{z},
\end{aligned}
$$

where $\mathbf{u}_{z}=\cos \theta \mathbf{u}_{r}-\sin \theta \mathbf{u}_{\theta}$. The unknown scattering amplitudes, $B_{0}, A_{1}, B_{1}$, and $A_{2}$, can be evaluated by applying the continuity of the tangential electric field and normal flux-density components, expressed in potential form, i.e.,

$$
\partial_{\theta} \Phi_{j}\left(r_{j}, \theta\right)=\partial_{\theta} \Phi_{j+1}\left(r_{j}, \theta\right)
$$

and

$$
\left.\varepsilon_{j} \partial_{r} \Phi_{j}(r, \theta)\right|_{r=r_{j+1}}=\left.\varepsilon_{j+1} \partial_{r} \Phi_{j+1}(r, \theta)\right|_{r=r_{j+1}},
$$

where $j=0,1$. This set of four linear equations can be compactly expressed in a matrix form $\mathbf{A} \bar{X}=\mathbf{b}$ for which the four unknowns are $\bar{X}=\left(B_{0} A_{1} B_{1} A_{2}\right)^{T}$. The system matrices read

$$
\mathbf{A}=\left(\begin{array}{cccc}
-\frac{1}{r_{1}^{2}} & A\left(r_{1}\right) & B\left(r_{1}\right) & 0 \\
\frac{2 \varepsilon_{h}}{r_{1}^{3}} & \varepsilon_{r}\left(r_{1}\right) A^{\prime}\left(r_{1}\right) & \varepsilon_{r}\left(r_{1}\right) B^{\prime}\left(r_{1}\right) & 0 \\
0 & A\left(r_{2}\right) & B\left(r_{2}\right) & -r_{2} \\
0 & \varepsilon_{r}\left(r_{2}\right) A^{\prime}\left(r_{2}\right) & \varepsilon_{r}\left(r_{2}\right) B^{\prime}\left(r_{2}\right) & -\varepsilon_{2}
\end{array}\right),
$$

and the excitation vector is

$$
\mathbf{b}=-E_{0}\left(\begin{array}{c}
r_{1} \\
\varepsilon_{h} \\
0 \\
0
\end{array}\right) .
$$

Note that primes denote the differentiation with respect to $r$. The determination of the scattering amplitudes at each region is reduced to a brute-force matrix inversion, an algebraically laborious but rather straightforward task.

After the modularization of the solution we can shift our focus on the available permittivity profiles. First, we consider the power profile that has been studied in [34]. Second, the formulated electrostatic differential equation of Eq. (6) can be solved for another family of radially inhomogeneous permittivity profiles, i.e., exhibiting exponential radial dependence of the type $e^{(n r)^{p}}$, with two parameters $n$ and $p$ ( $p$ is an integer). In this work, we focus on two particular exponential profiles, i.e., the linear $e^{n r}(p=1)$ (exp for short), and the inverse linear 
$e^{1 / n r}(p=-1)$ (inv exp). The term linear corresponds to the power of the exponent $n r$, and correspondingly its inverse $1 / n r$.

Exponential profiles can be applied for several purposes. For instance, the exponential profile can be approximated as $e^{(n r)^{p}} \approx 1+(n r)^{p}$ when $n r \rightarrow 0$ and $p \geq 0$. Assuming that the arbitrary constant $n$ can be of the form $b \omega_{p}^{2} / \omega^{2}$, where $b$ is a normalization length, the exponential profile gives an approximation of a Drude-like model, where the plasma frequency exhibits a radial dependence. The above observation can be generalized for every exponential profile, allowing the implementation of such profiles and also the modeling of realistic permittivity distributions $[39,40]$.

Similar forms of exponential permittivity profiles have been used as a phenomenological description for the problem of solution-solvent electrostatic interactions [41]. Here, we take the metamaterial or composite material approach and analyze exponential profiles from a lightscattering perspective. It is projected that these kinds of profiles can be used in an effective material description of artificially engineered composites or used as a modeling fit for an experimentally extracted scattering response, where the triggered mechanisms require a permittivity description beyond the standard stepwise homogenous model.

\section{A. Power-law profile}

We start our analysis by assuming a power-law profile, i.e.,

$$
\varepsilon_{r}(r)=\varepsilon_{1}\left(\frac{r}{b}\right)^{n},
$$

where $b$ is a normalization factor with units of meter and $n$ is the power factor $(n \in \mathbb{R})$. By inserting the above profile to the general ODE of Eq. (6) we obtain

$$
f^{\prime \prime}(r)+\frac{1}{r}(n+2) f(r)^{\prime}-\frac{2}{r^{2}} f(r)=0,
$$

known as the nonhomogeneous Euler-Cauchy differential equation, which has a solution of the form

$$
f(r)=A_{1} r^{p_{1}}+B_{1} r^{p_{2}}
$$

where

$$
p_{1,2}=-\frac{1}{2}\left[n+1 \mp \sqrt{(n+1)^{2}+8}\right],
$$

are the power factors of the solution. These factors exhibit certain interesting properties. For example, $p_{1}$ and $p_{2}$ are always of different sign for any value of $n$, and their product is constant, i.e.,

$$
p_{1} p_{2}=-2 \text {. }
$$

The limiting cases are $\lim _{n \rightarrow+\infty} p_{1}(n)=0$ and $\lim _{n \rightarrow-\infty} p_{1}(n)=+\infty$, while $p_{1}(0)=1$ and $p_{2}(0)=-2$ result in the well-known radial functions of a homogeneous permittivity.

\section{B. Linear exponential profile $\boldsymbol{e}^{\boldsymbol{n} r}$}

The first exponential profile under examination is the linear exponential profile, i.e.,

$$
\varepsilon_{r}(r)=\varepsilon_{1} e^{n \frac{r}{b}},
$$

where $n$ can be any arbitrary real parameter, and $b$ is a normalization radius with units $(\mathrm{m})$. For the sake of simplicity, $b$ is equal to the external radius $r_{1}$ and will be omitted.

Following Eq. (6), the formulated ODE is

$$
f^{\prime \prime}(r)+\frac{1}{r}(n r+2) f^{\prime}(r)-\frac{2}{r^{2}} f(r)=0,
$$

and its solution, expressed in terms of the corresponding $A(r)$ and $B(r)$ functions, reads

$$
A(r)=\frac{1}{n}\left(1-\frac{2}{n r}+\frac{2}{n^{2} r^{2}}\right),
$$

and

$$
B(r)=\frac{e^{-n r}}{r^{2}},
$$

where both $A(r)$ and $B(r)$ are singular at the origin. This is a rather counterintuitive fact since the profile (18) is smooth over the center of the sphere. In order to sidestep the effects of this behavior, a singularity subtracting core is required.

\section{Inverse-linear exponential profile $e^{1 / n r}$}

The second exponential case is the inverse-linear exponential profile (inv exp) expressed as

$$
\varepsilon_{r}(r)=\varepsilon_{1} e^{b / n r},
$$

with $n$ being again an arbitrary variable, similar to the exponential profile, and $b$ is the radius normalization factor, similar to the previous cases (for simplicity here we also assume $b=1$ ). This leads to

$$
f^{\prime \prime}(r)+\frac{1}{n r^{2}}(2 n r-1) f^{\prime}(r)-\frac{2}{r^{2}} f(r)=0,
$$

and the associated functions are

$$
A(r)=\frac{2 n r-1}{2 n-1}
$$


and

$$
B(r)=n \frac{2 n r+1}{2 n-1} e^{-\frac{1}{n r}},
$$

\section{Remarks about $A(r)$ and $B(r)$}

At this end, a couple of necessary observations about the character of $A(r)$ and $B(r)$ can be drawn. For both cases of homogeneous and power-law profiles $A(r)$ is a wellbehaving function at the origin (no singularity at $r=0$ ), while $B(r)$ contains a singularity, e.g., $r^{p_{1}}$ is smooth at the origin $\left(p_{1}>0\right.$ for every $\left.n\right)$, while $r^{p_{2}}$ is singular $\left(p_{2}<0\right.$ for every $n)$.

This observation, however, does not hold for every solvable case presented here. In particular, for the exp profile, both $A(r)$ and $B(r)$ [Eqs. (20) and (21)] are singular at the origin. In order to overcome this, a singularity extracting region at the origin (core) is therefore necessary for calculating the polarizability of the core-shell inclusion; the intact case can be approached as the limiting case of a vanishing core $\left(r_{2} \rightarrow 0\right)$. Note that the exp profile has no singularity at the origin.

On the other hand, for the inverse-exponential profile both $A(r)$ and $B(r)$ [Eqs. (24) and (25)] are smooth at the origin when $n \leq 0$, a rather counterintuitive result. This fact creates a rather unusual (in terms of standard boundary-value problems in electromagnetics) situation since both $A(r)$ and $B(r)$ satisfy the radial function within the bounded shell region. This gives a total of three unknown amplitudes $\left(B_{0}, A_{1}\right.$, and $\left.B_{1}\right)$, making the intact scatterer case (one interface) an underdetermined system. Here, we circumvent this by introducing an extra interface (core region) that completes the required conditions for the exact determination of the problem.

The inv exp permittivity profile is smooth at the center for $n \rightarrow \pm \infty$ since $\lim _{n \rightarrow \pm \infty} \varepsilon_{r}(r) \approx \varepsilon_{1}$. However, for the case when $n \rightarrow 0^{-}$we have either an epsilon-nearzero (ENZ) profile at the center $\left[\lim _{r \rightarrow 0^{-}} \varepsilon_{r}(r) \approx 0\right]$, or $\lim _{r \rightarrow 0^{+}} \varepsilon_{r}(r) \approx \infty$. The latter is a form of a perfect electric conductor- (PEC-)like behavior. Equivalently, the same limits can be reached when $r \rightarrow 0$ and $n<0$ and $r \rightarrow 0$ and $n>0$, i.e., ENZ and PEC behavior, respectively.

It is evident that these permittivity-induced peculiarities require the existence of a singularity regularization core region either as singularity subtraction or as a way to properly determine the formulated boundary-value scattering problem. Therefore, the consideration of a general coreshell setup is necessary for extracting a regular solution for both exponential profiles and analyzing the more general problem of a core-shell scatterer. The validity of our mathematical analysis is discussed in the Appendix, where the implementation of a multilayer approach is given and a comparison between the scattering spectrum is briefly discussed.

\section{ANALYSIS AND DISCUSSION}

Once the matrices A, and $\mathbf{b}$ are assembled (see Sec. II), all the unknown field amplitudes can be determined by the expression $\bar{X}=\mathbf{A}^{-1} \cdot \mathbf{b}$. The external amplitude $B_{0}$ represents the amplitude of the dipolar field created outside the scatterer, quantifying the dipole strength caused by the presence of an external field. In other words, parameter $B_{0}$ is nothing but the polarizability of the studied inclusion [42]. The resulting static polarizability can be computed as follows:

$$
\alpha_{s}=3 \frac{B_{0}}{r_{1}^{3} E_{0}}
$$

The extracted electrostatic polarizability quantifies only the electrostatic effects. This model successfully captures the radiation enhancement (or Fröhlich condition) without, however, taking into account any kind of radiation reaction that restores the conservation of energy for this physical system $[12,43,44]$. To do so, an imaginary term accounting for the radiation reaction needs to be introduced to restore the energy balance of this passive system. This new corrected [or modified long-wave approximation (MLWA) [44]] quasistatic polarizability reads $[45,46]$

$$
\alpha_{d}=\frac{-i(2 / 9) \alpha_{s} x^{3}}{1-i(2 / 9) \alpha_{s} x^{3}}
$$

where $x=k r_{1}$ is the size parameter relative to the host medium ( $k$ is the wavenumber of the host medium). Consequently, the scattering and extinction efficiencies are written as

$$
Q_{\mathrm{sca}}=\frac{6}{x^{2}}\left|\alpha_{d}\right|^{2}
$$

and

$$
Q_{\mathrm{ext}}=\frac{6}{x^{2}} \Re\left\{\alpha_{d}\right\}
$$

In the following sections all the scattering and extinction efficiencies depicted are given by Eq. (29), accounting also for the radiative reaction effects. In this sense, the validity of the proposed model expands beyond the electrostatic regime, and can be readily used up to moderate-sized spheres.

At this point it is necessary to refine the way the extracted dipole-scattering amplitude (polarizability) is expressed. The inversion of matrix (11) for a general $\varepsilon_{r}(r)$ profile can be compactly written as

$$
B_{0}^{\text {general }}=\frac{C \varepsilon_{r}\left(r_{1}\right)-\varepsilon_{0}}{C \varepsilon_{r}\left(r_{1}\right)+2 \varepsilon_{0}} E_{0} r_{1}^{3}
$$


where

$$
C=r_{1} \frac{\varepsilon_{2}\left[A^{\prime}\left(r_{1}\right) B\left(r_{2}\right)-A\left(r_{2}\right) B^{\prime}\left(r_{1}\right)\right]+\varepsilon_{r}\left(r_{2}\right) r_{2}\left[A^{\prime}\left(r_{2}\right) B^{\prime}\left(r_{1}\right)-A^{\prime}\left(r_{1}\right) B^{\prime}\left(r_{2}\right)\right]}{\varepsilon_{2}\left[A\left(r_{1}\right) B\left(r_{2}\right)-A\left(r_{2}\right) B\left(r_{1}\right)\right]+\varepsilon_{r}\left(r_{2}\right) r_{2}\left[A^{\prime}\left(r_{2}\right) B\left(r_{1}\right)-A\left(r_{1}\right) B^{\prime}\left(r_{2}\right)\right]}
$$

is the inhomogeneity factor. This complicated but rather straightforward expression conveys elegantly all the effects of the inhomogeneity in a core-shell structure.

The form of Eq. (30) facilitates the analysis of profiles that do not have an analytical solution, expanding its significance for inverse-scattering problems. For a given (arbitrary) material profile one can either experimentally or numerically extract the scattering spectrum of a given inhomogeneous sphere, and fit the phenomenological description of Eq. (31) to the observed spectrum by properly adjusting the inhomogeneity factor $C$. Therefore, the inhomogeneity factor is directly applicable for inverse-engineering and scattering purposes.

The case of an intact sphere can be approached by taking the limit of the inhomogeneity factor $C$ when $r_{2} \rightarrow 0$ in Eq. (31). Generally, this is a function of both $A(r)$ and $B(r)$. However, for the standard case of well-behaved functions, i.e., $\lim _{r \rightarrow 0} A(r)=0$ and $\lim _{r \rightarrow 0} B(r)=\infty$, the inhomogeneity factor includes only $A(r)$ and its value on the external boundary of the sphere, viz.,

$$
C=r_{1} \frac{A^{\prime}\left(r_{1}\right)}{A\left(r_{1}\right)}=\left.r_{1}[\ln (A(r))]^{\prime}\right|_{r=r_{1}} .
$$

The compact form of polarizability in Eq. (30) might also be of particular use for the plasmonic-scatteringenhancement case, since it generalizes the resonance condition in a simple manner, i.e.,

$$
\varepsilon_{r}\left(r_{1}\right)=-\frac{2 \varepsilon_{h}}{C}
$$

One can observe that the inhomogeneity factor contributes directly to the main resonant condition. Finally, when the radiative reaction is included [Eq. (27)], the resonance leads to the following complex expression:

$$
\varepsilon_{r}\left(r_{1}\right)=-\frac{2 \varepsilon_{h}}{C}-i \frac{2 \varepsilon_{h}}{C} x^{3},
$$

implying that the inhomogeneity factor modifies not only the resonant position but also the width and the maximum resonant absorption of plasmonic resonance $[45,46]$. This nontrivial fact enables the unprecedented control of the scattering process, hence, justifying why or how particular inhomogeneities (discrete or continuous) affect the scattering spectrum.
Exact forms of the extended quasistatic polarizability are of paramount importance for applications focused in controlling light-matter interactions, either as singlescattering effects or as collective effects in composite devices. For instance, the dispersion engineering problem can be approached by analytic, closed-form polarizability expressions; the effective medium description is proportional to the single-inclusion polarizability $[42,47]$.

Additionally, knowledge of the RI polarizability gives direct inspection for the limiting behavior of multilayer particles, especially when the number of layers increases. This limiting process obviously can lead to simpler and physically intuitive understanding regarding which parameters affect the scattering behavior of the proposed scatterer.

Actually, there is a remarkably large amount of modern applications, from optical antennas [48] to plasmonic devices and sensors [13], optical forces [49], and hot-electron photocatalysis $[15,50]$, where polarizability expressions give direct estimations on the scattering and absorptive characteristics of the corresponding systems. In all the above cases, engineered or stochastic (random) inhomogeneities of a single particle may significantly alter the overall scattering behavior. Therefore, closedform solutions of RI particles allow the direct study and implementation of these phenomena.

\section{A. Homogeneous core-shell: inhomogeneity perspective}

A core-shell particle with homogeneous regions (core $\varepsilon_{2}$, and shell, $\varepsilon_{1}$ ) is perhaps the simplest case of an inhomogeneous profile, with a stepwise function character. For this case $\left[A(r)=r\right.$ and $\left.B(r)=1 / r^{2}\right]$ the polarizability reads

$$
B_{0}^{\mathrm{cs}}=\frac{C \varepsilon_{1}-\varepsilon_{h}}{C \varepsilon_{1}+2 \varepsilon_{h}} E_{0} r_{1}^{3},
$$

where the introduced coefficient is

$$
C_{\mathrm{cs}}=-2+3 \frac{1}{1-\frac{\varepsilon_{2}-\varepsilon_{1}}{\varepsilon_{2}+2 \varepsilon_{1}} \eta^{3}},
$$

and $\eta=r_{2} / r_{1}$ is the radius ratio. The scaling factor $C$ approaches unity when either $\eta=0$ (no core) or the contrast between the core and shell permittivity is zero $\left(\varepsilon_{1}-\right.$ $\varepsilon_{2}=0$ ). On the other hand, $C=\varepsilon_{2} / \varepsilon_{1}$ for $\eta \rightarrow 1$, and 
the expression leads to the well-known polarizability for a core-shell sphere [51]

$$
B_{0}^{\mathrm{cs}}=\frac{\left(\varepsilon_{1}-\varepsilon_{h}\right)\left(\varepsilon_{2}+2 \varepsilon_{1}\right)+\eta^{3}\left(\varepsilon_{h}+2 \varepsilon_{1}\right)\left(\varepsilon_{2}-\varepsilon_{1}\right)}{\left(\varepsilon_{1}+2 \varepsilon_{h}\right)\left(\varepsilon_{2}+2 \varepsilon_{1}\right)+2 \eta^{3}\left(\varepsilon_{1}-\varepsilon_{h}\right)\left(\varepsilon_{2}-\varepsilon_{1}\right)} E_{0} r_{1}^{3} .
$$

The homogeneous core-shell particle has been used as a conceptual testbed for a great variety of applications, as stated above. A summary of the most general characteristics can be found in Refs. [51,52]. The response of such a sphere can be perceived as a combination between a solid sphere and a cavity where the overall scattering response is a result of the hybridization of the main plasmonic modes available for each structure [8]. Briefly, the position, width, and other qualitative characteristics are a function of all core-shell material and the radius ratio $\eta$.

\section{B. Power profile}

The polarizability of an intact sphere with a power profile has been given and studied to a certain extent in works such as Refs. [34,53]. Here we revisit these results, delivering additional insights regarding the scattering behavior of this resonant inhomogeneous sphere. The generalized polarizability of a core-shell inhomogeneous sphere with a power profile [Eq. (13)] reads

$$
B_{0}^{\text {power }}=\frac{C \varepsilon_{r}\left(r_{1}\right)-\varepsilon_{h}}{C \varepsilon_{r}\left(r_{1}\right)+2 \varepsilon_{h}}
$$

where the inhomogeneity factor is

$$
C_{\text {power }}=p_{2}+\frac{p_{1}-p_{2}}{1-\frac{\varepsilon_{2}-p_{1} \varepsilon_{r}\left(r_{2}\right)}{\varepsilon_{2}-p_{2} \varepsilon_{r}\left(r_{2}\right)} \eta^{p_{1}-p_{2}}} .
$$

Apparently, this result reduces to Eqs. (36) and (37) when $n=0, p_{1}=1, p_{1}=-2, \varepsilon_{r}\left(r_{1}\right)=\varepsilon_{1}$, and $\varepsilon_{r}\left(r_{2}\right)=$ $\varepsilon_{1}$. Inset Figs. 2(a) and 2(c) depict the scattering efficiency of such a sphere as a function of the permittivity $\varepsilon_{1}$ and the power factor $n$, for two distinctive cases, i.e., (a) $\eta=0.01$ and (b) $\eta=0.1$. We observe a smooth redshift of the main plasmonic resonance as the power factor increases.
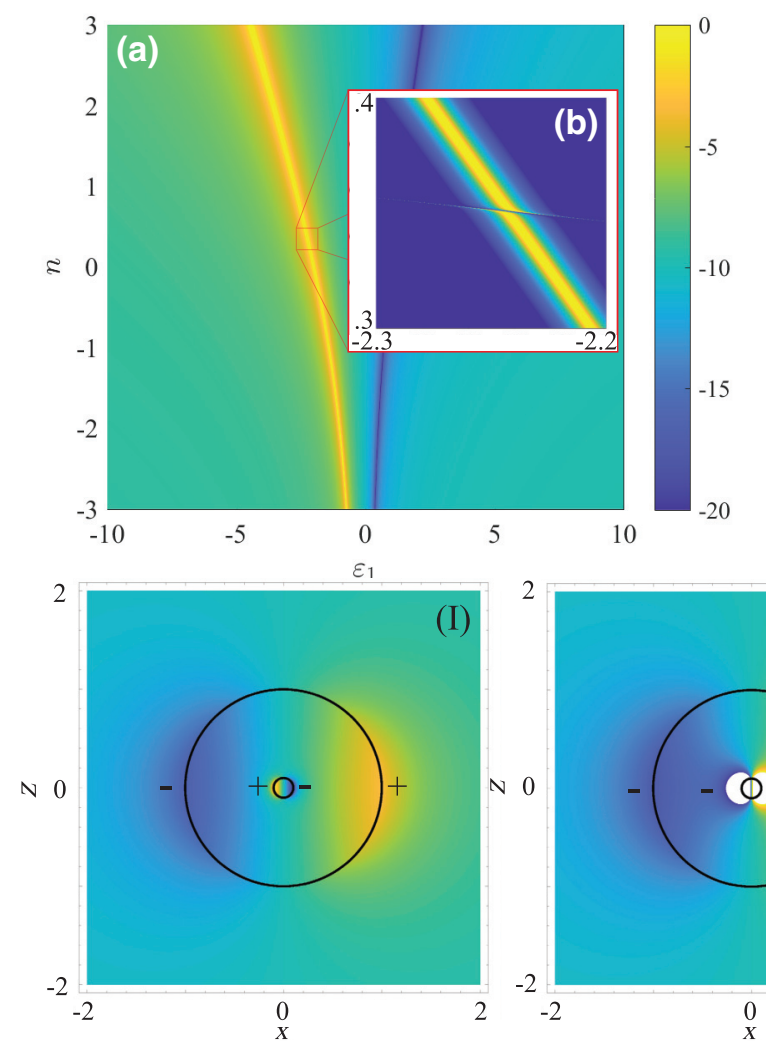

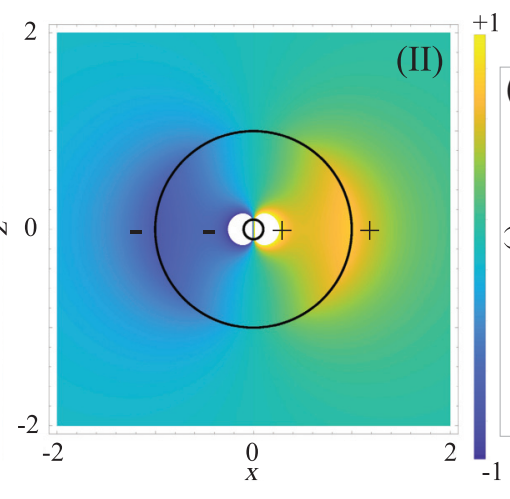

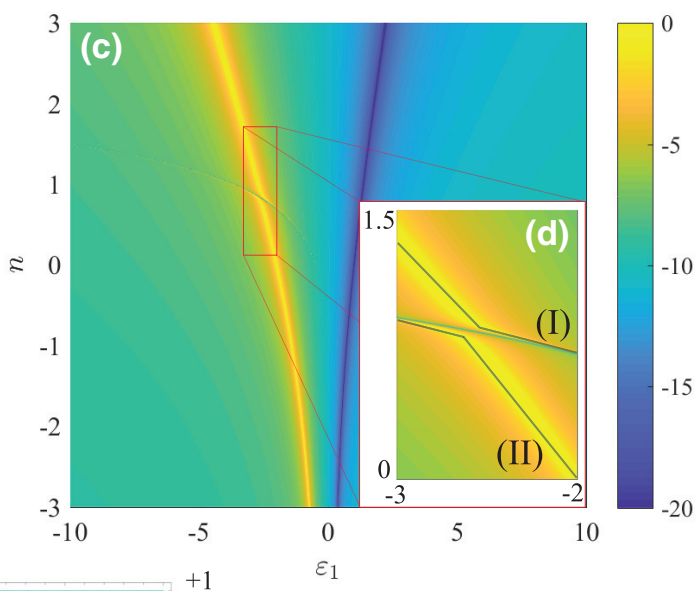

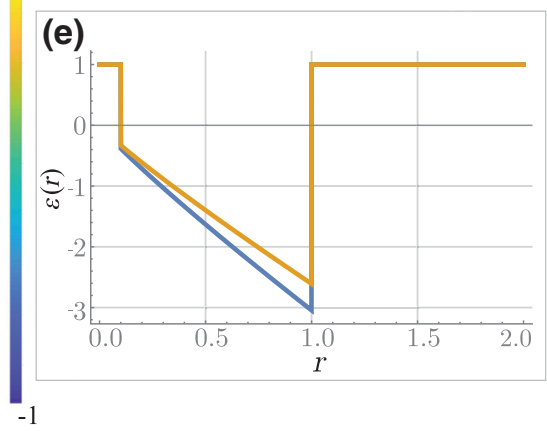

FIG. 2. The scattering efficiency $Q_{\text {sca }}$ (logarithmic scale) as a function of the power factor $n$, and permittivity $\varepsilon_{1}$ for (a),(b) $\eta=0.01$ (very small core radius) and (c),(d) $\eta=0.1$. In (a),(b),(c), and (d) the yellow regions indicate the radiation enhancement (plasmonic resonance) while the blue line indicates the scattering minimum. The insets in (b) and (d) depict a detail of the symmetric-antisymmetric degeneracy observed at the scattering spectrum. Figures (I) and (II) illustrate the (normalized) potential for the antisymmetric (I) and symmetric (II) resonances for the (d) case, i.e., $\eta=0$. . Finally, (e) depicts the permittivity profile for the case of $\eta=0.1, n=0.9$ at the antisymmetric resonance at $\varepsilon_{1}=-2.6087$ (orange curve) and the symmetric resonance (blue curve) at $\varepsilon_{1}=-3.045$, respectively. 
It is obvious that the power factor $n$ offers an extra degree of freedom for engineering the overall response. For example, the radius ratio dependence observed in Eq. (39) exhibits an exponent of the form $p_{1}-p_{2}=$ $\sqrt{(n+1)^{2}+8}$. This interesting feature reveals that the power-law profile plasmonic resonances with different fraction volume, $\eta^{p_{1}-p_{2}}$, than the volume dependence $\left(\eta^{3}\right)$ observed in a homogeneous core-shell case [see for example Eq. (37)] [52]. Note that for $n=-1$ this exponent obtains its global minimum value, i.e., $2 \sqrt{2}$.

Turning now to a simpler case, that of an intact inhomogeneous sphere $(\eta \rightarrow 0)$, the polarizability expression reduces to

$$
B_{0}=\frac{p_{1} \varepsilon_{r}\left(r_{1}\right)-\varepsilon_{h}}{p_{1} \varepsilon_{r}\left(r_{1}\right)+2 \varepsilon_{h}},
$$

since the inhomogeneity parameter is $C=p_{1}$. As can be seen, Eq. (40) exhibits resonant behavior when the condition $\varepsilon_{r}\left(r_{1}\right)=-\left(2 / p_{1}\right) \varepsilon_{h}$ is satisfied. For the limiting cases when $n \rightarrow+\infty$, the power factor is $p_{1} \rightarrow 0$, implying that $\varepsilon_{r}\left(r_{1}\right) \rightarrow-\infty$, while for $n \rightarrow-\infty$ we have $p_{1} \rightarrow+\infty$ and $\varepsilon_{r}\left(r_{1}\right) \rightarrow 0$. Similar trends can be deduced for the scattering minimum.

It is interesting to note that the existence of a core gives a zero-pole scattering crossing for values close to $n=0.36$ and $n=0.83$ for $\eta=0.01$ and $\eta=0.1$, as can be seen in Figs. 2(b) and 2(d). This point corresponds to a degenerate point between the symmetric and antisymmetric resonance. Specifically, the potential distribution for both antisymmetric (antibonding) and symmetric (bonding) resonances appear in inset Figs. 2(I) and 2(II). The antisymmetric resonance is characterized by a change of sign between the external (shell) and internal (core) resonances, as can be seen in Fig. 2(I). Equivalently, the internal and external potential distributions exhibit the same signs for the case of the symmetric one [Fig. 2(II)].

The plasmon hybridization model suggests that the resulting resonances of a core-shell structure are due to the contribution of two distinctive resonances, one of a solid sphere and its dual shape, i.e., a cavity surrounded by a metallic material [8]. In our case, the power-law sphere and cavity problem give two resonances approximately at $\varepsilon_{1}^{-}=-\left(2 / p_{1}\right) \varepsilon_{h}$ (symmetric, intact sphere) and $\varepsilon_{1}^{+}=-\left(p_{1} / 2 \eta^{n}\right) \varepsilon_{h}$ (antisymmetric, intact cavity), assuming that $r_{1}=1$ (normalized value) and $r_{2}=\eta r_{1}$. Requiring that both symmetric and antisymmetric resonances occur at the same point, i.e., $\varepsilon_{1}^{-}=\varepsilon_{1}^{+}$, we obtain the following expression:

$$
\eta=\left(\frac{p_{1}}{2}\right)^{2 / n}
$$

The extracted condition of Eq. (41) reveals the relation between the $n$ and the radius ratio $\eta$ for which a symmetricantisymmetric degeneracy occurs. Figure 3 illustrates the required $\eta$ as a function of the power factor. We observe

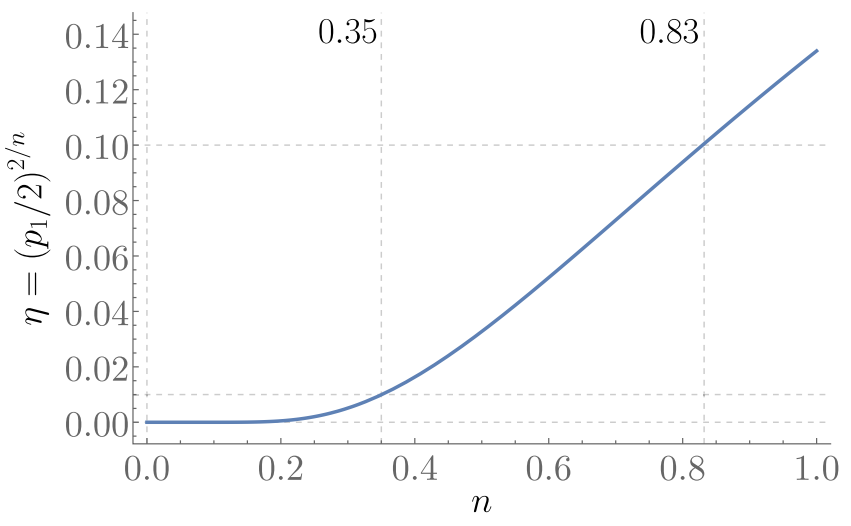

FIG. 3. Plot of Eq. (41), as a function of the power factor $n$. The plot validates the exact positions of the symmetric-antisymmetric degeneracy observed in Figs. 2 (a) and 2(c).

that even for the case of a vanishingly small power factor, i.e., an almost homogeneous profile, there is always a proper core-shell ratio that contributes to an observed degeneracy.

This observation may be the smoking gun for identifying the effects of inhomogeneity in realistically grown particles, since the symmetric and antisymmetric resonances exhibit several distinctively different scattering characteristics, such as the internal field distribution shown by the potential distribution of Figs. 2(I) and 2(II), or the position of the maximum absorption (see for example [52]). From an inverse-engineering perspective this mechanism reveals that even a small power-law material inhomogeneity can modify the antisymmetric resonance, forcing it to be strongly coupled to a trivial plane-wave excitation, even for the case of an extremely small core.

A similar type of symmetric-antisymmetric degeneracy occurs also in homogeneous core-shell spheres, this time only for vanishingly small cores and for a proper contrast between the core and host permittivities, i.e., $\varepsilon_{2}=4 \varepsilon_{h}$ for the case of a dipole-type resonances [52]. Here, however, the observed degeneracy is controlled by the power factor $n$ (for a fixed $\eta$ ), i.e., there is always a degeneracy point even for large core-shell ratios.

Moreover, as can be seen in Fig. 2(d), for values below the $n$, the symmetric resonance is dominant, while the antisymmetric exhibits very weak resonant coupling characteristics, i.e., vanishingly small resonant bandwidth and strength. Interestingly, this behavior is flipped for values above the branching power factor. In this case, the antisymmetric resonance dominates while the symmetric resonance exhibits weaker characteristics.

From a physical point of view, the symmetricantisymmetric plasmonic resonant degeneracy (crossing) creates a strong coupling regime, a rather attractive feature for systems where particularly strong light-matter interactions are required [54-57]. 
The observed form of resonant crossing is generally followed by an extremely sharp field enhancement at the core region, an indication that this structure can, in the ideal lossless case, support eigenmodes that allow almost infinite resonant lifetimes [7,58]. This type of degeneracy is attributed solely to the existence of a core to the system and the ability of tuning the resonances through the inhomogeneity factor.

At the vicinity of the center of the sphere the inhomogeneous permittivity shell exhibits an ENZ behavior allowing the extreme confinement of electric field. The ENZ shell behavior has been exploited for the conceptual design of scattering characteristics (radiative and nonradiative) for ordinary antennas [59], optical memories [60], and quantum sources [61]. Here the same behavior can be obtained, essentially affected by the inhomogeneity factor, enabling a different type of control over the embedded eigenmodes.

\section{Exponential profiles}

The analysis of the previous section can be repeated in a rigorous manner for the introduced exponential profiles. In an attempt to deliver some insights regarding the scattering peculiarities of such a sphere, we analyze the case of an intact sphere. As can be seen already from their mathematical treatment in Sec. II, these particular profiles require the existence of a regularization core region. Therefore, the case of an intact sphere and its polarizability can be approached by repeating the analysis of a core-shell structure and taking the limit case of a vanishingly small core $(\eta \rightarrow 0)$.

Starting with the linear exp profile, $e^{n r}$, the inhomogeneity factor of an intact sphere $(\eta \rightarrow 0)$ reduces to

$$
C_{\exp }=2 \frac{e^{n}(n-2)+n+2}{e^{n}\left(n^{2}-2 n+2\right)-2},
$$

where for $n \rightarrow 0$ we have $C_{\exp }=1$. Figure 4 depicts the plasmonic resonance spectrum at the parametric space of $\varepsilon_{1}$ and the factor $n$. One observes that in this case, large positive $n$ values shift both the scattering zero (Fig. 4 blue valley) and the pole (Fig. 5 yellow peak) toward the ENZ region. At the limit where $n \rightarrow+\infty$ both features (zero pole) finally cancel each other, creating an ultrasharp resonant degenerative point, a form of an embedded eigenstate [58]. However, negative $n$ values lead to strong shifts of both features towards opposite directions. Obviously, when $n=0$, the pole and the zero coincide with the homogeneous case, i.e., $\varepsilon_{1}=-2$ and $\varepsilon_{1}=1$, respectively.

This particular profile can be used for describing a scatterer that gradually desolves to the host medium. Requiring that $\varepsilon_{r}\left(r_{1}\right)=\varepsilon_{h}$ and $\varepsilon_{r}(0)=\varepsilon_{1}$ (where $\varepsilon_{1}$ can be any ordinary dispersive material) we obtain that

$$
n=\ln \frac{\varepsilon_{h}}{\varepsilon_{1}} .
$$

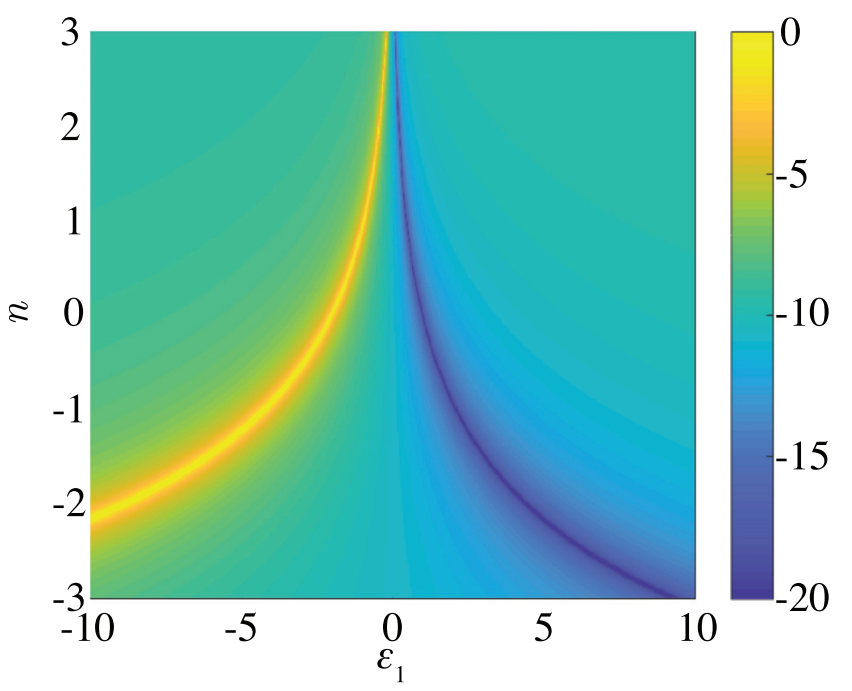

FIG. 4. Scattering efficiency $Q_{\text {sca }}$ (in logarithmic scale) for the linear exponential permittivity profile as a function of the scaling factor $n$ and the internal permittivity $\varepsilon_{1}$. Note that for large positive and large negative $n$ values both pole and zero converge or diverge, as a function of the parameter $\varepsilon_{1}$.

Therefore, for a given special value of $n$, the exponential profile can be used as a phenomenological description for a scatterer with smooth boundaries, such as ligand or irregular nanoparticles $[17,62]$ or for extremely small particles that electron density varies smoothly with respect to the radius (electron spill out) [63].

Note that Eq. (43) is valid for $\varepsilon_{1}>0$. In the case where $\varepsilon_{1}<0$, the expressions is generally complex with a value of $n=\ln \left|\varepsilon_{h} / \varepsilon_{1}\right|+i \pi$, and the material description

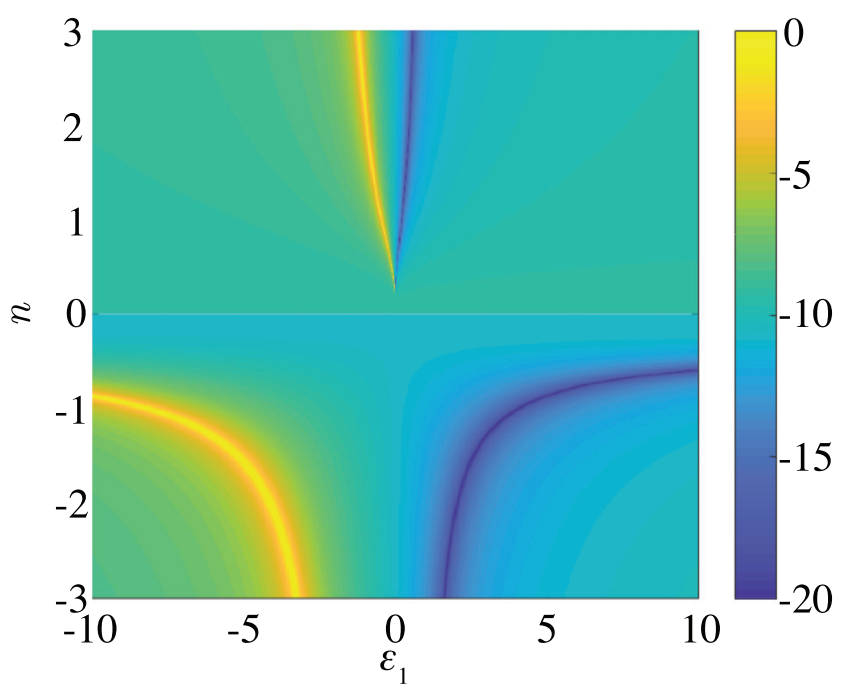

FIG. 5. Scattering efficiency $Q_{\text {sca }}$ (in logarithmic scale) of the inverse-linear exponential permittivity profile as a function of the scaling factor $n$ and the internal permittivity $\varepsilon_{1}$. Note the pole-zero scattering degeneracy occur for $n \rightarrow 0^{+}$, indicating an extreme embedded mode. 
is intrinsically complex. In this work, we focus only on the first approach of such profiles, and hence a thorough analysis of the impact of a complex power factor $n$ is left for future studies.

Finally, the inverse linear exponential profile gives an inhomogeneity factor of the form

$$
C_{\text {inv-exp }}= \begin{cases}1+\frac{1}{n}-\frac{1}{2 n+1}, & n>0 \\ \frac{2 n}{2 n-1}, & n \leq 0 .\end{cases}
$$

In this case, the distribution of the inhomogeneity factor $C_{\text {inv-exp }}$ depends on the sign of the parameter $n$. The corresponding spectrum of the inv exp case (Fig 5) reveals a reverse trend with respect to the exp case. The homogeneous-profile case with the resonance close to $\varepsilon_{1}=$ -2 and scattering minimum at $\varepsilon_{1}=1$, can be asymptotically reached for $n \rightarrow \pm \infty$, as can be seen in Fig. 5 . On the other hand, small negative values of $n$ cause an extreme shift of the observed resonance, while the case of small positive values of $n$ result in pole-zero degeneracy at the ENZ limit, akin to the $n \rightarrow+\infty$ for the exp case.

At this rather exotic condition, the core of the inv exp sphere exhibits a PEC behavior that exponentially transits to an ENZ behavior. Note that the same degeneracy is observed for both exponential cases and constitutes an effectively new type of degeneracy, in contrast to the ones reported in $[7,58,59]$. The main difference here comes from the fact that the reported embedded eigenmodes require a step-homogeneous (discrete) permittivity profile where one of the interfaces approaches ENZ values. In this work, the existence of a resonant degeneracy is predicted this time for a continuously inhomogeneous permittivity profile. In particular, for the limit where the degeneracy is observed, e.g., when $n \rightarrow 0^{+}$for the inv exp profile, the permittivity experiences extreme values (in the order of $10^{200}$ or more for $n<1 / 2$ ) towards the inner regions, effectively imitating the hard-wall ENZ trapping requirements $[7,58]$. In other words, our findings suggest that a continuously inhomogeneous profile could, conceptually, support embedded eigenmodes. As a final remark, these kinds of inhomogeneities can, in principle, induce a symmetric-antisymmetric degeneracy, similar to the power-law profile. The key feature for this is the study of a corresponding core-shell problem, as described in the previous sections.

\section{CONCLUSIONS}

The expression of Eq. (30) generalized the concept of the homogeneous polarizability, allowing us to rigorously explore the nontrivial physical mechanisms for a whole new family of graded-index particles. The introduced polarizability description offers a direct homogenization formula for the effective permittivity perspectives in both a realistic and engineered manner [64]. Additionally, this description can be used to reverse engineer the inhomogeneity coefficient $C$, fitting to experimental or numerical data. In this way, the experimentally observed deviations of the plasmonic resonances on deeply subwavelength spheres [40] can potentially find simpler phenomenological interpretation; the same general form of polarizability can be extrapolated even for nonanalytically solvable profiles, allowing an approximative estimation regarding their behavior.

In particular, the cases of a power-law, an exponential, and an inverse-exponential permittivity profile have been rigorously studied, through the derived exact polarizability expressions. The analysis of these structures reveals a wealth of nontrivial plasmonic scattering characteristics, such as the existence of a scattering degeneracy between the symmetric-antisymmetric resonances that can be potentially used as an identification of small inhomogeneities, or for applications where a strong coupling resonant regime is required. Additionally, an exotic case of an embedded eigenmode existent in the inv exp profile has been identified, i.e., near an exponential a PEC(core)-to-ENZ(shell) transition. It is evident that the introduced exact polarizability model is a key concept for the direct inspection of realistic and other conceptual inhomogeneity-induced effects.

Aside from the above cases, the analytical study can be implemented for a wide range of applications where rigorous modeling of artificially grown inhomogeneous structures, e.g., stratified spheres, transformation optics [65], irregularly shaped particles [62], is required. In brief, the proposed inhomogeneously refined model can directly replace the widely used homogeneous polarizability description for each of the aforementioned cases.

The same analysis can also be deployed for thermoplasmonic and mechanic-plasmonic applications, where the existence of a temperature and pressure gradient changes the permittivity distribution, causing an effective inhomogeneous profile. These thermally induced inhomogeneities might lead to improved heat-assisted magnetic recording [66] and reinforce our deeper understanding of heat-diffusion problems in plasmonic nanoparticles [67].

Lastly, diffusive effects, especially between interfaces that follow a radially dependent distribution can be easily approached by the introduced models, for example, in heavily doped semiconductor scatterers offering a plethora of new absorption and scattering functionalities [68]. In conclusion, it is envisioned that the presented study will stimulate novel energy control and harvesting ideas for nanophotonic applications, such as the implementation of subwavelength plasmonic particles exhibiting Luneburg, Eaton, or more exotic graded-index profiles. 


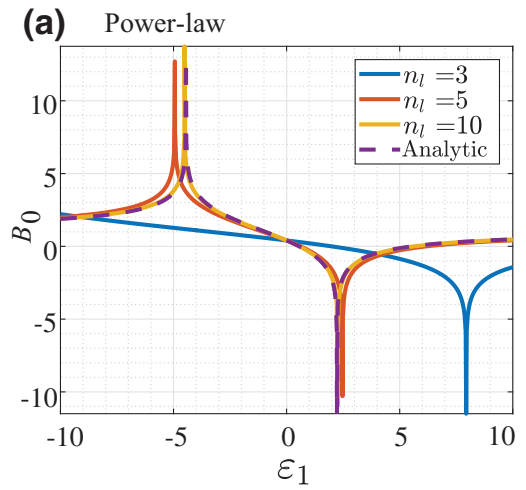

(b) Exp

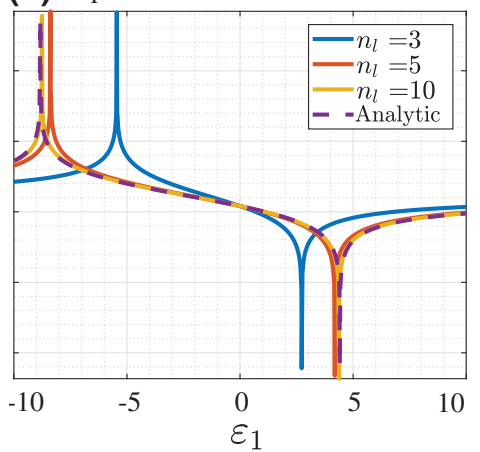

(c) Inv exp

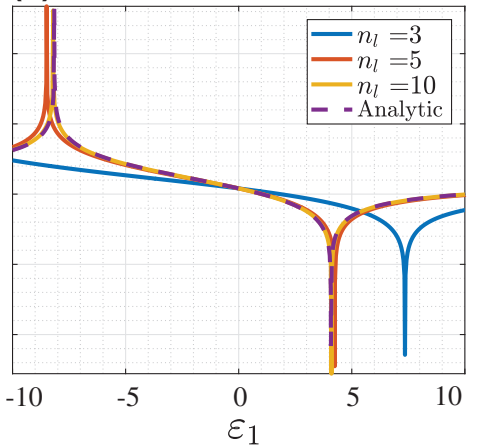

FIG. 6. The scattering amplitude $B_{0}$ (colors in adjusted logarithmic scale) for all three analytical cases (dashed purple lines) compared with the response of a multilayered sphere of $n_{l}=3$ (blue lines), $n_{l}=5$ (red lines), and $n_{l}=10$ (orange lines) layers. Subfigures depict (a) the power profile for $n=2$, (b) the exponential profile for $n=-2$, and (c) the inv-exp profile for $n=-1$. A multilayer sphere with more than $n_{l}=10$ can accurately capture the scattering trends, verifying in this way the validity of the theoretical analysis.

\section{ACKNOWLEDGMENTS}

The work is supported by the Aalto University ELEC Doctoral School Scholarship. D.C.T thanks Professor Nader Engheta for being the host at the University of Pennsylvania, where parts of this work have been completed.

\section{APPENDIX A: VALIDATING THE ANALYTICAL MODELS}

An available check point regarding the validity of the mathematical analysis presented in Sec. II can be derived by comparing the extracted polarizability results with a step-homogeneous multilayered sphere. The polarizability of such a multilayered sphere can be enumerated following an iterative analysis presented in [35]. The permittivity of each layer exhibits a constant value, extracted from the continuous profile. In this sense, the permittivity profile of the multilayered sphere is nothing but the discretized version of each of the aforementioned continuous permittivity profiles.

Figure 6 depicts a comparison for the three cases, as a function of the number of layers. As we can see, a multilayered structure with more than $n_{l}=10$ layers reproduces the scattering behavior very accurately. In the computations, the permittivity of layers is taken as the geometrical mean value between the external and internal radius of each layer.

It is evident that the analytical models introduced previously give sound and accurate predictions versus the multilayer approach. Indeed, the main scattering characteristics are successfully captured, as can be seen from Fig. 6, especially regarding the shift of the main resonant characteristics. However, as stated in the introductory section, the multilayer iterative approach offers little physical insights on the studied problem. Specifically, all the qualitative results extracted for the power-law, exp, and inv exp profiles became obvious only through the introduced analytical models. In particular, the inhomogeneity factor $C$ conveys, in an simple manner, the exact scattering behavior at the studied parametric space (permittivity $\varepsilon_{1}$ versus factor $n$ ). Therefore, the present analytical study can shed light on inhomogeneity-induced scattering peculiarities, cultivating fertile grounds for the exploitation of RI scatterers for engineering purposes, physical phenomenology, and computational benchmarking.

[1] Robert A. Shore, Scattering of an electromagnetic linearly polarized plane wave by a multilayered sphere: Obtaining a computational form of Mie coefficients for the scattered field, IEEE Antennas Propag. Mag. 57, 69 (2015).

[2] Matthew E. Stewart, Christopher R. Anderton, Lucas B. Thompson, Joana Maria, Stephen K. Gray, John A. Rogers, and Ralph G. Nuzzo, Nanostructured plasmonic sensors, Chem. Rev. 108, 494 (2008).

[3] Alex Krasnok, Martín Caldarola, Nicolas Bonod, and Andrea Alú, Spectroscopy and biosensing with optically resonant dielectric nanostructures, Adv. Opt. Mater. 6, 1701094 (2018).

[4] Harry A. Atwater and Albert Polman, Plasmonics for improved photovoltaic devices, Nat. Mater. 9, 205 (2010).

[5] Andrea Alù and Nader Engheta, Achieving transparency with plasmonic and metamaterial coatings, Phys. Rev. E 72, 016623 (2005).

[6] Zhichao Ruan and Shanhui Fan, Superscattering of Light from Subwavelength Nanostructures, Phys. Rev. Lett. 105, 013901 (2010).

[7] Mário G. Silveirinha, Trapping light in open plasmonic nanostructures, Phys. Rev. A 89, 023813 (2014).

[8] Emil Prodan, Corey Radloff, Naomi J. Halas, and Peter Nordlander, A hybridization model for the plasmon response of complex nanostructures, Science 302, 419 (2003).

[9] Boris Luk'yanchuk, Nikolay I. Zheludev, Stefan A. Maier, Naomi J. Halas, Peter Nordlander, Harald Giessen, and 
Chong Tow Chong, The Fano resonance in plasmonic nanostructures and metamaterials, Nat. Mater. 9, 707 (2010).

[10] Xiaofeng Fan, Weitao Zheng, and David J. Singh, Light scattering and surface plasmons on small spherical particles, Light Sci. Appl. 3, e179 (2014).

[11] Craig F. Bohren and Donald R. Huffman, Absorption and Scattering of Light by Small Particles (John Wiley \& Sons, New York, 2008).

[12] Pedro de Vries, David V. van Coevorden, and Ad Lagendijk, Point scatterers for classical waves, Rev. Mod. Phys. 70, 447 (1998).

[13] K. Lance Kelly, Eduardo Coronado, Lin Lin Zhao, and George C. Schatz, The optical properties of metal nanoparticles: The influence of size, shape, and dielectric environment, J. Phys. Chem. B 107, 668 (2003).

[14] Antoine Bricard, Jean-Baptiste Caussin, Nicolas Desreumaux, Olivier Dauchot, and Denis Bartolo, Emergence of macroscopic directed motion in populations of motile colloids, Nature 503, 95 (2013).

[15] Alejandro Manjavacas, Jun G. Liu, Vikram Kulkarni, and Peter Nordlander, Plasmon-induced hot carriers in metallic nanoparticles, ACS Nano 8, 7630 (2014).

[16] Jin Cai, James P. Townsend, Tom C. Dodson, Paul A. Heiney, and Alison M. Sweeney, Eye patches: Protein assembly of index-gradient squid lenses, Science 357, 564 (2017).

[17] Sheng Peng, Jeffrey M. McMahon, George C. Schatz, Stephen K. Gray, and Yugang Sun, Reversing the sizedependence of surface plasmon resonances, Proc. Natl. Acad. Sci. 107, 14530 (2010).

[18] Thomas Zentgraf, Yongmin Liu, Maiken H. Mikkelsen, Jason Valentine, and Xiang Zhang, Plasmonic Luneburg and Eaton lenses, Nat. Nanotechnol. 6, 151 (2011).

[19] Rudolf Karl Luneburg and Max Herzberger, Mathematical Theory of Optics (University of California Press, Los Angeles, 1964).

[20] Chen-To Tai, Maxwell fish-eye treated by Maxwell equations, Nature 182, 1600 (1958).

[21] Juan C. Miñano, Perfect imaging in a homogeneous threedimensional region, Opt. Express 14, 9627 (2006).

[22] Arthur L. Aden and Milton Kerker, Scattering of electromagnetic waves from two concentric spheres, J. Appl. Phys. 22, 1242 (1951).

[23] James R. Wait, Electromagnetic scattering from a radially inhomogeneous sphere, Appl. Sci. Res. Sect. B 10, 441 (1962).

[24] Nader Engheta and Charles H. Papas, Ambartsumian's principle of invariance and the reflection of radio waves from plane inhomogeneous slabs, Appl. Phys. B Photophysics Laser Chem. 30, 183 (1983).

[25] Bruce R. Johnson, Invariant imbedding $T$ matrix approach to electromagnetic scattering, Appl. Opt. 27, 4861 (1988).

[26] Bruce R. Johnson, Exact theory of electromagnetic scattering by a heterogeneous multilayer sphere in the infinitelayer limit: Effective-media approach, J. Opt. Soc. Am. A 16, 845 (1999).

[27] Alexander Shalashov and Egor Gospodchikov, Simple approach to electromagnetic scattering by small radially inhomogeneous spheres, IEEE Trans. Antennas Propag. 64, 3960 (2016).
[28] Georgios D. Kolezas and Grigorios P. Zouros, CFVIE formulation for EM scattering on inhomogeneous anisotropicmetallic objects, IEEE Trans. Antennas Propag. 65, 3788 (2017).

[29] Milton Kerker, The Scattering of Light and Other Electromagnetic Radiation: Physical Chemistry: A Series of Monographs (Academic Press, New York, 2013), Vol. 16.

[30] Victor A. Babenko, Ludmila G. Astafyeva, and Vladimir N. Kuz'min, Electromagnetic Scattering in Disperse Media: Inhomogeneous and Anisotropic Particles (Springer Science \& Business Media, Berlin, 2003).

[31] Chen-To Tai, The electromagnetic theory of the spherical Luneberg lens, Appl. Sci. Res. Sect. B 7, 113 (1959).

[32] B. S. Westcott, Exact solutions for electromagnetic wave propagation in spherically stratified isotropic media, Math. Proc. Cambridge Philos. Soc. 64, 227 (1968).

[33] Chen-To Tai, Some wave functions and potential functions pertaining to spherically stratified media, J. Res. Natl. Bur. Stand. D. Radio Propag. 67D, 199 (1963).

[34] L. Dong, G. Q. Gu, and K. W. Yu, First-principles approach to dielectric response of graded spherical particles, Phys. Rev. B 67, 224205 (2003).

[35] Ari Sihvola and Ismo V. Lindell, Transmission line analogy for calculating the effective permittivity of mixtures with spherical multilayer scatterers, J. Electromagn. Waves Appl. 2, 741 (1988).

[36] Vincent Laquerbe, Romain Pascaud, Thierry Callegari, Laurent Liard, and Olivier Pascal, Analytical model to study the electrostatic resonance of subwavelength radially inhomogeneous negative permittivity spheres, IEEE Antennas Wirel. Propag. Lett. 16, 2894 (2017).

[37] David Colton and Rainer Kress, Inverse Acoustic and Electromagnetic Scattering Theory (Springer Science \& Business Media, New Jersey, 2012), Vol. 93.

[38] J. David Jackson, Classical Electrodynamics (Wiley Online Library, New York, 1975).

[39] Stig Lundqvist and Norman H. March, Theory of the Inhomogeneous Electron Gas (Springer Science \& Business Media, New York, 2013).

[40] Thomas Christensen, Wei Yan, Søren Raza, Antti-Pekka Jauho, N. Asger Mortensen, and Martijn Wubs, Nonlocal response of metallic nanospheres probed by light, electrons, and atoms, ACS Nano 8, 1745 (2014).

[41] Edward W. Castner, Graham R. Fleming, Biman Bagchi, and Mark Maroncelli, The dynamics of polar solvation: Inhomogeneous dielectric continuum models, J. Chem. Phys. 89, 3519 (1988).

[42] Ari Sihvola, Electromagnetic Mixing Formulas and Applications (IET, London, 1999), Vol. 47.

[43] Rémi Carminati, Jean-Jacques Greffet, Carsten Henkel, and J. M. Vigoureux, Radiative and non-radiative decay of a single molecule close to a metallic nanoparticle, Opt. Commun. 261, 368 (2006).

[44] Alexander Moroz, Non-radiative decay of a dipole emitter close to a metallic nanoparticle: Importance of higher-order multipole contributions, Opt. Commun. 283, 2277 (2010).

[45] Sergei Tretyakov, Maximizing absorption and scattering by dipole particles, Plasmonics 9, 935 (2014).

[46] Dimitrios C. Tzarouchis, Pasi Ylä-Oijala, and Ari Sihvola, Unveiling the scattering behavior of small spheres, Phys. Rev. B 94, 140301(R) (2016). 
[47] Reehyang Kim, Kyungjae Chung, Ju Young Kim, Yunyong Nam, Sang-Hee Ko Park, and Jonghwa Shin, Metal nanoparticle array as a tunable refractive index material over broad visible and infrared wavelengths, ACS Photonics 5, 1188 (2018).

[48] Palash Bharadwaj, Bradley Deutsch, and Lukas Novotny, Optical antennas, Adv. Opt. Photonics 1, 438 (2009).

[49] Dongliang Gao, Andrey Novitsky, Tianhang Zhang, Fook Chiong Cheong, Lei Gao, Chwee Teck Lim, Boris Luk'yanchuk, and Cheng-Wei Qiu, Unveiling the correlation between non-diffracting tractor beam and its singularity in Poynting vector, Laser Photon. Rev. 9, 75 (2015).

[50] Mark L. Brongersma, Naomi J. Halas, and Peter Nordlander, Plasmon-induced hot carrier science and technology, Nat. Nanotechnol. 10, 25 (2015).

[51] Dimitrios Tzarouchis and Ari Sihvola, Light scattering by a dielectric sphere: Perspectives on the Mie resonances, Appl. Sci. 8, 184 (2018).

[52] Dimitrios C. Tzarouchis and Ari Sihvola, General scattering characteristics of resonant core-shell spheres, IEEE Trans. Antennas Propag. 66, 323 (2018).

[53] Victor Y. Reshetnyak, Igor P. Pinkevych, Timothy J. Sluckin, and Dean R. Evans, Cloaking by shells with radially inhomogeneous anisotropic permittivity, Opt. Express 24, A21 (2016).

[54] Andrea E. Schlather, Nicolas Large, Alexander S. Urban, Peter Nordlander, and Naomi J. Halas, Near-field mediated plexcitonic coupling and giant rabi splitting in individual metallic dimers, Nano Lett. 13, 3281 (2013).

[55] Tomasz J. Antosiewicz, S. Peter Apell, and Timur Shegai, Plasmon-exciton interactions in a core-shell geometry: From enhanced absorption to strong coupling, ACS Photonics 1, 454 (2014).

[56] Gülis Zengin, Martin Wersäll, Sara Nilsson, Tomasz J. Antosiewicz, Mikael Käll, Timur Shegai, Realizing Strong Light-Matter Interactions between Single-Nanoparticle Plasmons and Molecular Excitons at Ambient Conditions, Phys. Rev. Lett. 114, 157401 (2015).

[57] Kotni Santhosh, Ora Bitton, Lev Chuntonov, and Gilad Haran, Vacuum Rabi splitting in a plasmonic cavity at the single quantum emitter limit, Nat. Commun. 7, 11823 (2016).

[58] Francesco Monticone and Andrea Alù, Embedded Photonic Eigenvalues in 3D Nanostructures, Phys. Rev. Lett. 112, 213903 (2014).

[59] Aycan Erentok and Richard W. Ziolkowski, A hybrid optimization method to analyze metamaterial-based electrically small antennas, IEEE Trans. Antennas Propag. 55, 731 (2007).

[60] Sylvain Lannebère and Mário G. Silveirinha, Optical metaatom for localization of light with quantized energy, Nat. Commun. 6, 8766 (2015).

[61] Iñigo Liberal and Nader Engheta, Nonradiating and radiating modes excited by quantum emitters in open epsilonnear-zero cavities, Sci. Adv. 2, e1600987 (2016).

[62] R. Carmina Monreal, S. Peter Apell, and Tomasz J. Antosiewicz, Infrared absorption and hot electron production in low-electron-density nanospheres: A look at real systems, J. Phys. Chem. Lett. 8, 524 (2017).

[63] Søren Raza, Nicolas Stenger, Shima Kadkhodazadeh, Søren V. Fischer, Natalie Kostesha, Antti Pekka Jauho, Andrew Burrows, Martijn Wubs, and N. Asger Mortensen, Blueshift of the surface plasmon resonance in silver nanoparticles studied with EELS, Nanophotonics 2, 131 (2013).

[64] Uday K. Chettiar and Nader Engheta, Internal homogenization: Effective permittivity of a coated sphere, Opt. Express 20, 22976 (2012).

[65] Ashkan Vakil and Nader Engheta, Transformation optics using graphene, Science 332, 1291 (2011).

[66] Svetlana V. Boriskina, Thomas Alan Cooper, Lingping Zeng, George Ni, Jonathan K. Tong, Yoichiro Tsurimaki, Yi Huang, Laureen Meroueh, Gerald Mahan, and Gang Chen, Losses in plasmonics: From mitigating energy dissipation to embracing loss-enabled functionalities, Adv. Opt. Photonics 9, 775 (2017).

[67] Boris S. Luk'Yanchuk, Andrey E. Miroshnichenko, Michael I. Tribelsky, Yuri S. Kivshar, and Alexei R. Khokhlov, Paradoxes in laser heating of plasmonic nanoparticles, New J. Phys. 14, 093022 (2012).

[68] Jacob B. Khurgin, Relative merits of phononics vs. plasmonics: The energy balance approach, Nanophotonics 7, 305 (2018). 